\title{
Logarithm of a Function, a Well-Posed Inverse Problem
}

\author{
Silvia Reyes Mora, Víctor A. Cruz Barriguete, Denisse Guzmán Aguilar \\ Instituto de Física y Matemáticas, Universidad Tecnológica de la Mixteca, Huajuapan de León, Oax, México \\ Email: sreyes@mixteco.utm.mx
}

Received November 13, 2013; revised December 13, 2013; accepted December 20, 2013

Copyright (c) 2014 Silvia Reyes Mora et al. This is an open access article distributed under the Creative Commons Attribution License, which permits unrestricted use, distribution, and reproduction in any medium, provided the original work is properly cited. In accordance of the Creative Commons Attribution License all Copyrights @ 2014 are reserved for SCIRP and the owner of the intellectual property Silvia Reyes Mora et al. All Copyright (c) 2014 are guarded by law and by SCIRP as a guardian.

\begin{abstract}
It poses the inverse problem that consists in finding the logarithm of a function. It shows that when the function is holomorphic in a simply connected domain $\Omega \subseteq \mathbb{C}$, the solution at the inverse problem exists and is unique if a branch of the logarithm is fixed. In addition, it's demonstrated that when the function is continuous in a domain $\Omega \subseteq X$, where $X$ is Hausdorff space and connected by paths. The solution of the problem exists and is unique if a branch of the logarithm is fixed and is stable; for what in this case, the inverse problem turns out to be well-posed.
\end{abstract}

\section{KEYWORDS}

Logarithm Function; Inverse Problem; Stability

\section{Introduction}

The inverse problems generally are ill-posed in Hadamard sense. These words lead us to think that there exist inverse problems that are well-posed and which are possible to be solved analytically [1].

The ill-posed problems do not fulfill with at least one of the conditions of existence, uniqueness or stability of the solution. Nevertheless, if a problem does not have a solution, or the solution is not unique, it is possible to correct in spite of doing considerations on the domain and the co-domain of the operator who represents the problem.

Unlike a complex number different from zero for which, it is always possible to find his logarithm. The functions need certain conditions on his domain to guarantee the existence of his logarithm [2]. It appears that one of these conditions is that the domain is simply connected. To assure the uniqueness of the logarithm of a function, it is essential to take a branch of the logarithm.

In this paper, there appears the problem of finding the logarithm of a given function, by different techniques. It is demonstrated that the above-mentioned problem is an inverse stable problem in Hadamard sense [3]. Therefore, the problem of existence and uniqueness is solved. In addition, it is demonstrated that it is stable, which transforms it into a well-posed inverse problem. It is realized the analysis of the solution of the inverse problem for when the domain of the inverse operator, it corresponds to the functions that are not annulled and in addition they are holomorphic in some region [4]. It is demonstrated that when $\Omega \subseteq \mathbb{C}$ is a simply connected domain, the solution of the inverse problem exists and is unique. In a similar way, when the space $Y$, it corresponds to the space of the continuous functions that are not annulled on a region $\Omega \subseteq X$ simply connected and where $Z$ is a Hausdorff's space and connected for paths, the solution of the inverse problem exists, is unique and is stable [5].

\section{Exposition of the Problem}

It's known that not all the real numbers have logarithm, nevertheless, all complex numbers have it.

The natural question that arises is under what conditions a function has logarithm. To answer to the previous question, an operator $A: X \rightarrow Y$ is considered, if $A=\exp$, and $X, Y$ are spaces of functions, it is possible 
to pose the direct problem: From a function on $f \in X$, find to a function $g \in Y$ such that $g=\exp (f)$. The corresponding inverse problem is given a function $g \in Y$, to find to a function $f \in X$, such that $g=\exp (f)$. It's known that the inverse of the operator exp is the operator log, so the inverse problem can be seen as the search of the logarithm of a given function. Notice that as is considered the operator $A=\exp$, it has felt to take as space $Y$ to the functions that are not annulled and in addition be continuous or holomorphic on some region; nevertheless they must determine the conditions that the region must fulfill to demonstrate the existence, uniqueness and stability of the inverse problem.

\section{Holomorphic Logarithm}

In this section, it is considered that $X$ corresponds to the holomorphic functions and $Y$ to the holomorphic functions that are not annulled on any region. There's demonstrated that when $\Omega$ is a simply connected domain, the solution of the inverse problem exists and is unique when a branch of the logarithm is fixed. The problem consists of knowing if given a function holomorphic $f$ can be a function $g \in Y$, such that $f=\exp (g)$.

Definition: Given a domain $\Omega \subseteq \mathbb{C}$, and be $f: \Omega \rightarrow C^{*}$ and $g: \Omega \rightarrow C$. It is said that $g$ is a logarithm of $f$ if $f=\exp (g)$. If the functions $f$ and $g$ are holomorphic in $\Omega$, it is said that $g$ is a holomorphic logarithm of $f$ [6].

Theorem 1: Let exp: $H(\Omega) \rightarrow H^{*}(\Omega)$ be, where $\Omega$ is a simply connected set of the plane, then the solution of the inverse problem exists and is unique.

Proof: If it thinks that $\Omega$ it is a simply connected domain, by theorem of the Riemann application, the simply connected domains of the flat sound of two types: the conformal equivalent to the plane $\mathbb{C}$ and the conformal equivalent to the unitary disc $D$. For such a motive, the existence of the solution of the inverse problem, are obtained as a consequence of the propositions 1, 2 and 3 . As for the uniqueness, it is essential to take a branch of the logarithm.

Proposition 1: Every function $f \in H^{*}(D)$ admits a holomorphic logarithm.

Proof: It is known that $f(z) \neq 0$ for every $z \in D$. Let $w_{0}$ be a point such that $\exp \left(w_{0}\right)=f\left(z_{0}\right)$. It's defined by:

$$
\psi(z)=w_{0}+\int_{\gamma_{z}} \frac{f^{\prime}(\xi)}{f(\xi)} \mathrm{d} \xi
$$

where $\gamma_{z}$ is the curve that joins to $z$ with $z_{0} . f$ it's holomorphic and is not annulled on $D$, then $\frac{f^{\prime}}{f}$ is holomorphic on $D$. Then, $\int_{\gamma_{z}} \frac{f^{\prime}(\xi)}{f(\xi)} \mathrm{d} \xi$ it's definite as well. This way, $\psi^{\prime}(z)=\frac{f^{\prime}(z)}{f(z)}$. Consider

$$
h(z)=\frac{\exp (\psi(z))}{f(z)} \text {, then } h^{\prime}(z)=\frac{f(z) \exp (\psi(z)) \psi^{\prime}(z)-f^{\prime}(z) \exp (\psi(z))}{f^{2}(z)}=0
$$

$\forall z \in D$. Then, $h(z)$ it is constant on $D$. So, $h\left(z_{0}\right)=\frac{\exp \left(\psi\left(z_{0}\right)\right)}{f\left(z_{0}\right)}=\frac{\exp \left(w_{0}\right)}{w_{0}}=1$.

Then $f(z)=\exp (\psi(z))$.

Let $g(z)=\psi(z)-w_{0}+\log \left(w_{0}\right)$ be, then $f(z)=\exp (g(z))$ and the proof it is complete.

Proposition 2: Let $\Omega$ be a simply connected domain own of $\mathbb{C}$, admits a holomorphic logarithm.

Proof: Consider $f \in H^{*}(\Omega)$, since $\Omega$ be a simply connected domain own of $\mathbb{C}$, there is an conformal function $g: D \rightarrow \Omega$, such that $f \circ g \in H(D)$. Since $f \in H^{*}(\Omega)$, follows that $f \circ g \in H^{*}(D)$. By the proposition 1 , the function $f \circ g$ admits a holomorphic logarithm in $D$. That is, there exists $h: D \rightarrow \mathbb{C}$ such that $f \circ g(w)=\exp (h(w))$.

Hereby $f(z)=\exp \left(h \circ g^{-1}\right)(z)$ and therefore $f$ admits a holomorphic logarithm in $\Omega$.

Proposition 3: Every function $f \in H^{*}(\mathbb{C})$ admits a holomorphic logarithm.

Proof: Since $f$ it is not annulled in $\mathbb{C}$ and $f^{\prime} \in H(\mathbb{C})$. Then $\frac{f^{\prime}(z)}{f(z)} \in H(\mathbb{C})$, that is, there exist a 
function $F \in H(\mathbb{C})$ such that is the primitive of $\frac{f^{\prime}(z)}{f(z)}$, that is to say, $F^{\prime}=\frac{f^{\prime}(z)}{f(z)}$ for everything $z \in \mathbb{C}$.

Notice that

$$
(f \exp (-F))^{\prime}(z)=f^{\prime} \exp (-F(z))-f(z) F^{\prime}(z) \exp (-F(z))=\left(f^{\prime}(z)-f(z) F^{\prime}(z)\right) \exp (-F(z))=0 .
$$

Then $f(z) \exp (-F(z))=C$, if $C=\exp (k)$ it follows that $f=\exp (k+F)$.

\section{Continuous Logarithm}

In this section, the inverse problem it's studied, when the condition weakens of being a holomorphic function to being a continuous function. Nevertheless, on having asked him only continuity to the functions and having tried to solve the inverse problem, it is needed to do more restrictions on the domain of the above mentioned functions.

It is considered to be that $X=C(\Omega)$ and the space $Y=C^{*}(\Omega)$ that correspond to the spaces of continuous and continuous that are not annulled function respectively. There is demonstrated that when $\Omega \subseteq Z$ it's a simply connected domain and $Z$ is Hausdorff space and connected by paths, the solution of the inverse problem exists, is unique if a branch of the logarithm is fixed adapted and is stable.

Theorem 2 Let exp:C $(\Omega) \rightarrow C^{*}(\Omega)$ be, where $\Omega$ a simply connected set is (not necessarily it is a subset of the plane) content in Hausdorff space and connected by paths; then the solution of the inverse problem exists, is unique and is stable.

Proof: The existence of the solution of the inverse problem, it's demonstrated in the proposition 3 and the existence in the proposition 4 .

Since exp:C $(\Omega) \rightarrow C^{*}(\Omega)$, where $C(\Omega)$ and $C^{*}(\Omega)$ are Banach spaces, in addition, the operator is continuous; then, by the open mapping theorem, the inverse operator is continuous and therefore, the solution of the inverse problem is stable.

Definition: Let $\tilde{X}$ and $Y$ be topological spaces. Consider to $p: \tilde{X} \rightarrow Y$ a continuous function. Is say that $p$ is a covering map if $\forall y \in Y$, it exists a neighborhood $U$ for $y$, with following properties:

1) $p^{-1}(U)=\bigcup_{j \in J} \tilde{U}_{j}$ with $\tilde{U}_{k} \cap \tilde{U}_{l}=\varnothing \forall k \neq l$.

2) $\tilde{U}_{j}$ is a neighborhood for $\tilde{X}$ such that $\left.p\right|_{\tilde{U}_{j}}$ is a homeomorphism on $U, \forall j \in J$.

Proposition 4: The exp : $\mathbb{C} \rightarrow \mathbb{C}^{*}$ function is a covering map.

Definition: Let $f: X \rightarrow Y$ be a continuous function and let $p: \tilde{X} \rightarrow Y$ be a covering map. The $g$ application is a lifting for $f$ (in respect of $p$ ) if $(p \circ g)=f$.

Note that if it is known that the exp function is a covering map, then to define the lifting, and under the conditions for the existence and uniqueness of the lifting, it will be had that $($ expo $g)=f$; and therefore on having demonstrated the existence and uniqueness of the lifting, there will be demonstrated the existence and uniqueness of the logarithm of a continuous function .

\subsection{Uniqueness of the Lifting}

For the uniqueness of the lifting it is necessary that the $X$ topological space be connected and Hausdorff.

Theorem 3 Let $p: \tilde{X} \rightarrow Y$ be a local homeomorphism, let $X$ be a connected and Hausdorff space and let $f: X \rightarrow Y$ be a continuous application. Suppose that $g_{1}$ and $g_{2}$ are lifting of $f$. Then, if $x_{0} \in X$ exists, such that $g_{1}\left(x_{0}\right)=g_{2}\left(x_{0}\right)$, it follows that $g_{1}=g_{2}$ in $X$.

Proof: The set $E=\left\{x \in X: g_{1}(x)=g_{2}(x)\right\}$ is defined. Note that $x_{0} \in E$ and therefore $E \neq \varnothing$. If it is shown that $E$ is open and closed it follow that $E=X$. To show that $E$ is a closed set, enough to prove that $X \backslash E$ is an open set. Let $y \in X \backslash E$ be, clearly $f_{1}(y) \neq f_{2}(y)$ and since $X$ is a Hausdorff space, exist neighborhoods $V_{f_{1}(y)} \neq V_{f_{2}(y)}$ for $f_{1}(y)$ and $f_{2}(y)$, respectively such that $V_{f_{1}(y)} \cap V_{f_{2}(y)}=\varnothing$. Consider that $W=f_{1}^{-1}\left(V_{f_{1}(y)}\right) \cap f_{2}^{-1}\left(V_{f_{2}(y)}\right)$, note that $W$ isn't an empty set, then $y \in W$, so, $W$ is open set since is a finite intersection of open sets. Let $x \in W$ be, then $f_{1}(x) \in V_{f_{1}(y)}$ and $f_{2}(x) \in V_{f_{2}(y)}$. What implies that $f_{1}(x) \neq f_{2}(y)$. Therefore $W \subset X \backslash E$ and so, the $X \backslash E$ open set and then the $E$ set is closed. To show that $E$ is an open set. Let $x \in E$ be and $\tilde{a}=f_{1}(x)=f_{2}(x)$. By hypothesis, there is a neighborhood $\tilde{U}$ for 
$\tilde{a}$ such that $p(\tilde{U})=U$ is open set and $\left.p\right|_{\tilde{U}}$ is an homeomorphism. Since $f_{1}$ and $f_{2}$ are continuous function, there is $V$ an neighborhood for $y$ such that $f_{i}(V) \backslash \tilde{U}$ and $p\left(f_{1}(v)\right)=f(v)=p\left(f_{2}(v)\right)$. Since $\left.p\right|_{\tilde{U}}$ is an injective function, it follows that $f_{1}(v)=f_{2}(v)$ so, $V \backslash E$ it's a closed set and then the $E$ set is open. Since $Y$ is connected space and $E$ is a non-empty, open and closed set, it follows that $E=Y$.

\subsection{Existence of the Lifting}

The study of the existence of the lifting needs of the study of the fundamental group.

Let $\alpha_{k}: I \rightarrow X$ be, where $k=1,2$, curves that begin and end in $x$, it is to say, they are closed curves. Is said that $\alpha_{1}$ it is related with $\alpha_{2}, \alpha_{1} \sim \alpha_{2}$, if it exists $H: I \times I \rightarrow X$ a continuous function such that

$$
H(0, t)=\alpha_{1}(t), H(1, t)=\alpha_{2}(t), H(s, 0)=H(s, 1)=x
$$

The $H$ function is called continuous homotopy.

It is easy to see that the relation $\sim$ is an equivalence relation. Since it is known well, everything equivalence relation induces a partition. In this case the classes $[\alpha]$ are formed by the set of curves $\beta$ that are homotopic to $\alpha$. Intuitively it is possible to define the operation join curved and the above mentioned operation gives a structure of group.

Definition: The first group of homotopy of $X$ is defined, with basis $x \in X$ :

$$
\pi_{1}(X, x)=\{[\alpha]\},
$$

where $\alpha$ is a closed curve.

We noted that $\tilde{\alpha}$ is the curve $\alpha(-t)$.

The following result gives necessary and sufficient conditions for the existence of the lifting.

Theorem 4: Let $p:(\tilde{X}, x) \rightarrow\left(Y, y_{0}\right)$ be a covering map and let $f:\left(X, x_{0}\right) \rightarrow\left(Y, y_{0}\right)$ be a continuous function, with $X$ a connected by paths set. Then they are equivalent:

1) A lifting $g:\left(X, x_{0}\right) \rightarrow\left(\tilde{X}, \tilde{x}_{0}\right)$ there exists for $f$;

2) $f *\left(\pi_{1}\left(X, x_{0}\right)\right) \subset p *\left(\pi_{1}\left(X, x_{0}\right)\right)$.

Where $g^{*}$ is the induced mapping of fundamental groups.

Proof: The demonstration 1) $\Rightarrow 2$ ) obviously, because $f *=p * \tilde{f} *$.

There will be demonstrated that 2) $\Rightarrow 1$ ). Let $x \in X$ be and let $\gamma$ be a curve in $X$ from $x_{0}$ to $x$. The curve $f_{\gamma}$ in $Y$ that it begins in $y_{0}$ it has a unique lifting $\tilde{f}_{\gamma}$ that it begins $\tilde{x}_{0} \cdot \tilde{f}(x)=\tilde{f}_{\gamma}(1)$ is defined. It is demonstrated that it is definite as well, independently of the choice of $\gamma$, let $\gamma^{\prime}$ be another curve from $x_{0}$ to $X$. Then $\left(f_{\gamma^{\prime}}\right) \bullet\left(\tilde{f}_{\gamma}\right)$ is a closed curve of $h_{0}$ to $y_{0}$ with:

$$
\left[h_{0}\right] \in f *\left(\pi_{1}\left(X, x_{0}\right)\right) \subset p *\left(\pi_{1}\left(\tilde{X}, \tilde{x}_{0}\right)\right) .
$$

This means that there is a homotopy $h_{t}$ from $h_{0}$ to a closed curve $h_{1}$ that gets up to a closed curve $\tilde{h}_{1}$ in $\tilde{X}$ based in $\tilde{x}_{0}$. Property homotopy covering is applied to $h_{t}$ to obtain a lifting $\tilde{h}_{t}$. Since $\tilde{h}_{1}$ is a closed curve to $\tilde{x}_{0}, h_{0}$ it's too. By the uniqueness of the lift curve, the first half of $\tilde{h}_{0}$ is $\tilde{f}_{\gamma^{\prime}}$ and the second half is $\tilde{f}_{\gamma}$ route the other way around, with the common midpoint $\tilde{f}_{\gamma}(1)=\tilde{f}_{\gamma^{\prime}}(1)$. This shows that $\tilde{f}_{\gamma}$ it's definite as well. Need to show that $\tilde{f}$ is a continuous function. Let $U \subset Y$ be a neighborhood of $f(x)$ that it has a lifting $\tilde{U} \subset \tilde{X}$ containing to $\tilde{f}(x)$ such that $p: \tilde{U} \rightarrow U$ is an homeomorphism. A connected by path neighborhood $V$ is chosen for $x$ with $f(V) \subset U$. The curves from $x_{0}$ to $x^{\prime} \in V$ points, it is possible to take a given fixed curve $\gamma$ from $x_{0}$ to $x$ follow by curves $\eta$ in $V$ from $y$ to the points $y^{\prime}$. Then, the curves $\left(f_{\gamma}\right) \bullet\left(f_{\eta}\right)$ in $Y$ it has lifting $\left(\tilde{f}_{\gamma}\right) \bullet\left(\tilde{f}_{\eta}\right)$ where $\tilde{f}_{\eta}=p^{-1} f_{\eta}$ and $p: U \rightarrow \tilde{U}$ is the inverse of $p: \tilde{U} \rightarrow U$. So, $\tilde{f}(V) \subset \tilde{U}$ and $\left.\tilde{f}\right|_{V}=p^{-1} \tilde{f}$, therefore $\tilde{f}$ is continuous in $X$.

\section{REFERENCES}

[1] C. W. Groetsch, "Inverse Problems: Activities for Undergraduates,” The Mathematical Association of America, Ohio, 1999.

[2] A. Browder, “Topology in the Complex Plane,” The American Mathematical Monthly, Vol. 107 No. 10, 2006, pp. 393-401. https:/getinfo.de/app/Topology-in-the-Complex-Plane/id/BLSE\%3ARN079983226

[3] A. Hatcher, “Algebraic Topology,” Cambridge University Press, Cambridge, 2009. 
[4] L. V. Ahlfors, “Complex Analysis,” McGraw-Hill, New York, 1979.

[5] A. Kirsch, “An Introduction to the Mathematical Theory of Inverse Problems," 2nd Edition, Springer, Berlin, 2011. http://dx.doi.org/10.1007/978-1-4419-8474-6

[6] E. Stein and R. Shakarchi, “Complex Analysis,” Princeton University Press, Princeton, 2009. 